\title{
Effects of On-Line Vortex Cooling on the Microstructure and Mechanical Properties of Wire Arc Additively Manufactured Al-Mg Alloy
}

\author{
Baoxing Wang ${ }^{1}$, Guang Yang ${ }^{1, *}$, Siyu Zhou ${ }^{1, *}$, Can Cui ${ }^{2}$ and Lanyun Qin ${ }^{1}$ \\ 1 Key Laboratory of Fundamental Science for National Defense of Aeronautical Digital Manufacturing \\ Process, Shenyang Aerospace University, Shenyang 110136, China; bx.w@foxmail.com (B.W.); \\ qinly@sau.edu.cn (L.Q.) \\ 2 Avic Shenyang Aircraft Design and Research Inatitute, Shenyang 110136, China; cuican1978@163.com \\ * Correspondence: yangguang@sau.edu.cn (G.Y.); zhousiyu@sau.edu.cn (S.Z.); Tel.: +86-180-4003-7100 (G.Y.); \\ +86-138-9865-4801 (S.Z.)
}

Received: 27 June 2020; Accepted: 22 July 2020; Published: 27 July 2020

\begin{abstract}
A novel on-line vortex cooling powered by low-cost compressed air was proposed to reduce common defects such as low forming precision, coarse grains, and pores caused by heat accumulation in the Wire Arc Additive Manufacturing (WAAM) of aluminum alloy. The impacts of interlayer cooling (IC), substrate cooling (SC), on-line cooling (OL), and natural cooling (NC) processes were compared on the morphology, microstructure, and mechanical properties of as-deposited walls, revealing that the OL process significantly lowers the interlayer temperature and improves forming precision. The high cooling rate produced by the OL process reduced the absorption of hydrogen in the molten pool, lowering porosity. Furthermore, the grains are refined due to the developed undercooling. However, the high cooling rate enhanced the segregation potential of $\mathrm{Mg}$ element and raised the content of the $\beta$ phase. Conclusively, the maximum tensile strength, elongation, and microhardness of the as-deposited wall are achieved via the OL process, and the fine-grain strengthening mechanism plays an important role in improving mechanical properties. The OL process is cheaper and poses a significant effect; it is highly suitable for the additive manufacturing of complex components compared with other forced cooling processes.
\end{abstract}

Keywords: wire arc additive manufacturing (WAAM); on-line vortex cooling; microstructure and mechanical properties

\section{Introduction}

The newly introduced digital additive manufacturing technology works by depositing metal parts in layerwise [1]. Since it is less costly and saves time [2], many people have increasingly regarded additive manufacturing as a solution to meet personalized customization. In recent years, compared with Laser Depositing Manufacturing (LDM) [3] and Selective Laser Melting (SLM) [4], Wire Arc Additive Manufacturing (WAAM) has attracted far more attention in the field of additive manufacturing, for example, in the aerospace field $[5,6]$. This is attributed to its lower initial cost, higher forming efficiency, and higher material utilization $[5,6]$.

With the rapid advancement of WAAM, a wide range of studies on WAAM aluminum alloy have been initiated, where a variety of aluminum alloy wires have been adopted as the feeding materials of WAAM, Al-Mg alloys, among others [7]. In particular, Al-Mg alloys are employed in aerospace because they resist corrosion and have efficient welding properties. However, the melting point of aluminum alloy is low $\left(\sim 660^{\circ} \mathrm{C}\right)$, but its fluidity is satisfactory and it is easily overheated between 
layers during the additive process. This results in poor forming precision, coarse structure, degradative property, and occurrence of pores.

A study by Derekar et al. [8] on the influence of different interpass temperatures through controlling the substrates preheated temperatures, 50 and $100{ }^{\circ} \mathrm{C}$ for wall- 1 and wall-2, revealed that the deposits had increased number of pores at high interpass temperature. Ren et al. [9] studied interpass cooling $\left(200,160,120\right.$ and $\left.80^{\circ} \mathrm{C}\right)$ of Al-6.3Mg alloy which adopted the cold metal transfer advanced process during WAAM process. The results demonstrate that reducing the interpass temperature effectively improved the mechanical properties and reduced the difference between the horizontal and vertical properties of the deposits. Besides, Geng et al. [10] studied the influence of different interpass temperatures $\left(50,80,120\right.$ and $\left.150^{\circ} \mathrm{C}\right)$, whereby they found that extreme high temperatures caused inconsistent layer cross-sectional dimension during deposition. With the difficulty of continuous forming of the aluminum alloy due to thermal accumulation, the control of heat dissipation is a scheme to ensure the depositing quality of WAAM. Relevant scholars have conducted some research on this drawback. For example, Henckell et al. [11] used a mixture of nitrogen and hydrogen at a flow rate of $30 \mathrm{~L} / \mathrm{min}$ to continuously cool the surface of the deposits during S355 steel depositing to enhance the heat dissipation ability and improve the forming ability of WAAM. The cooling nitrogen and hydrogen are expensive but do not refrigerate effectively in a flow rate of only $30 \mathrm{~L} / \mathrm{min}$; thus, the property of the deposits improved unsatisfactorily. Henckell et al. [12] also studied the energy input in WAAM with Gas Metal Arc Welding (GMAW), whereby they reduced welding energy input to lead to an adaptation of geometrical and microstructural features of additively manufactured work pieces. Su et al. [13] adopted WAAM to deposit Al-Mg alloy, which, based on cold metal transfer (CMT) technology, is where they used to manufacture Al-Mg alloys with different heat inputs by adjusting wire feed speed (WFS) and travel speed (TS). However, properties show no significant change with different TS. Wu et al. [14,15] adopted gas tungsten arc welding based WAAM (GT-WAAM) depositing TC4, where they set a gas cover on the surface of the deposits and achieved an accelerated deposition efficiency and a reduction in the oxidation of titanium alloy. Another study by Li et al. [16] assessed the influence of different cooling processes on geometric accuracy using a thermoelectric cooling device to cool the wall. Usually, the thermoelectric cooling device relies on conduction heat dissipation, and though the cooling effect in their experiment was satisfactory, the flexibility and adaptability were poor, it was unsuitable for different deposits accumulated layer by layer.

In this study, to refine the grain, ensure the forming quality and improve the forming efficiency, vortex tube and water-cooling substrate were performed in WAAM. The geometric shape, microstructure, and mechanical properties of deposits were investigated. We analyzed the influence on the results and assessed the reliability of the control WAAM.

\section{Experiment}

\subsection{Experiment Setup}

The apparatus comprised a FUNIUS TPS4000 power source and a welding torch (FUNIUS Inc., Pettenbach, Austria), water cooling unit (SHENY Inc., Shenyang, China), and welding robot (KUKA Inc., Augsburg, Germany), as shown in Figure 1. An infrared thermometer was used to measure the interlayer temperature between the deposition of layers. The feeding material used in this study included ER5356 aluminium alloy welding wire with a diameter of $1.2 \mathrm{~mm}$, deposited on a 5052 aluminium alloy substrate with dimensions of $200 \mathrm{~mm} \times 200 \mathrm{~mm} \times 20 \mathrm{~mm}$. The chemical compositions of feeding wire and structure are highlighted in Table 1. 


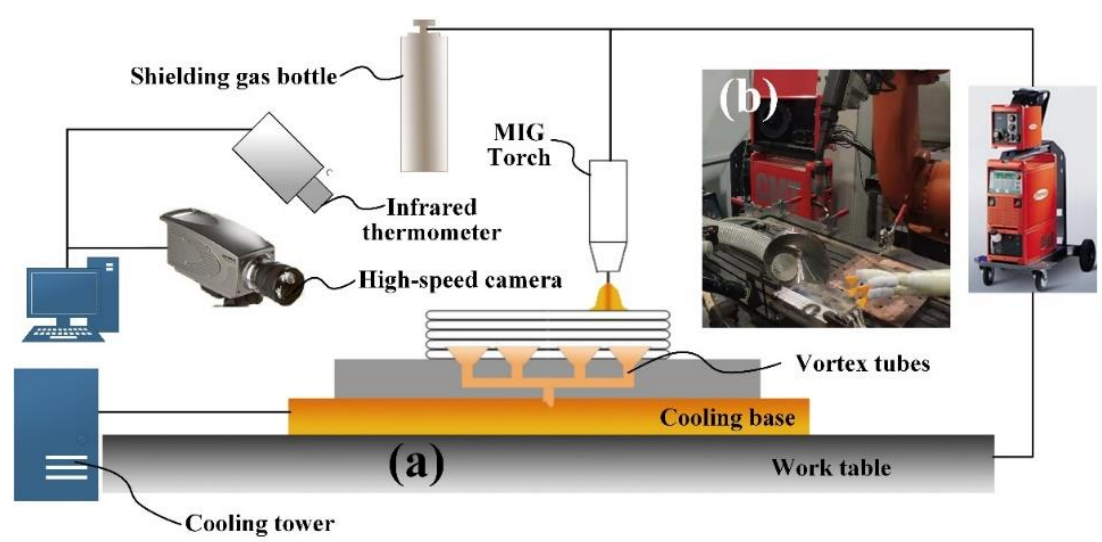

Figure 1. Wire Arc Additive Manufacturing (WAAM) deposition system: (a) schematic diagram, (b) physical diagram.

Table 1. Chemical composition of ER5356 and 5052Al (wt. \%).

\begin{tabular}{cccccccc}
\hline Composition & $\mathbf{M g}$ & $\mathbf{M n}$ & $\mathbf{C r}$ & $\mathbf{C u}$ & $\mathbf{Z n}$ & $\mathbf{F e}+\mathbf{S i}$ & $\mathrm{Al}$ \\
\hline ER5356 & $4.5-5.5$ & $0.05-0.2$ & $0.15-0.35$ & 0.1 & 0.1 & 0.5 & Bal. \\
5052 & $2.2-2.8$ & 0.1 & $0.05-0.2$ & 0.1 & 0.1 & 0.45 & Bal. \\
\hline
\end{tabular}

Four straight wall structures were as-deposited at interlayer time $90 \mathrm{~s}$ using the WAAM system with four different cooling processes. The welding parameters are shown in Table 2. Welding-grade argon (99.995\% purity) was applied as the shielding gas for the WAAM torch.

Table 2. Process parameters for WAAM deposition.

\begin{tabular}{ccc}
\hline Process Parameters & Details & Parameter \\
\hline \multirow{2}{*}{ Deposition power } & Current & $160 \mathrm{~A}$ \\
& Arc voltage & $23 \mathrm{~V}$ \\
Speed & Travel speed & $0.022 \mathrm{~m} / \mathrm{s}$ \\
& Wire feed speed & $11 \mathrm{~m} / \mathrm{min}$ \\
Distance and angle & Contact tube to workpiece & $12 \mathrm{~mm}$ \\
Flow rate(argon) & filler wire to workpiece & $90^{\circ}$ \\
Deposition power setting & WAAM torch & $24 \mathrm{~L} / \mathrm{min}$ \\
\hline
\end{tabular}

\subsection{Fast Cooling System}

To evaluate the effectiveness of the fast cooling system, a commercially available vortex tube and a water-cooling base were fitted to the WAAM torch as shown in Figure 2. With an air compressor (YOUMAN Inc., Shanghai, China), a stable pressure-air stream was delivered in the vortex tube, and the high pressure airflow in the vortex tube generated heat transfer, instantly producing low-pressure low-temperature gas, as the low-pressure high-temperature gas is ejected from both ends of the vortex tube. The vortex tube parameters are highlighted in Table 3. 


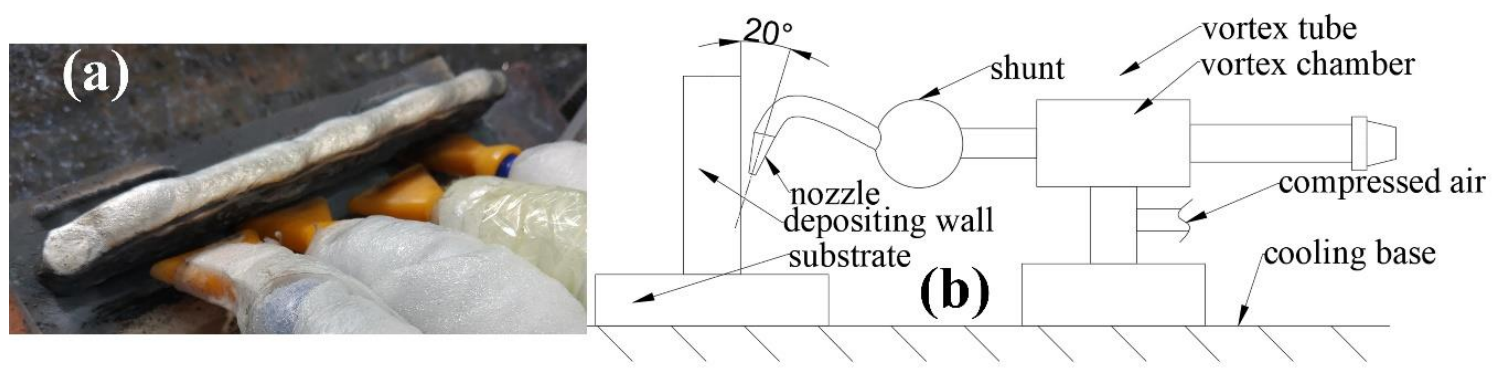

Figure 2. Vortex cooling system: (a) physical diagram, (b) schematic diagram.

Table 3. Process parameters of the vortex tube.

\begin{tabular}{cccc}
\hline $\begin{array}{c}\text { Parameters } \\
\text { Unit }\end{array}$ & $\begin{array}{c}\text { Air Pressure } \\
\text { Bar }\end{array}$ & $\begin{array}{c}\text { Gas Consumption } \\
\text { Stard Liter Per Minute (SLPM) }\end{array}$ & $\begin{array}{c}\text { Cooling Capacity } \\
\text { Kcal/hr }\end{array}$ \\
\hline Details & 6.9 & 1410 & 857 \\
\hline
\end{tabular}

Cooling air was oriented to the side of the as-deposited wall while the stable $20{ }^{\circ} \mathrm{C}$ water flowed through the water-cooled constant. Four cooling processes were specifically evaluated, that is, turning on the vortex tube after the complete deposition of each layer (interlayer cooling (IC)), keeping the vortex tube opened during the deposition process (on-line cooling (OL)), keeping the cooling water flowing inside the water-cooled constant during the deposition process (substrate cooling (SC)), depositing with natural cooling (NC). Whilst the compressed air enters the vortex chamber, the compressed air at room temperature transforms into high-temperature gas and cooling gas. The cooling gas is transferred to the shunt. The shunt fractionates the cooling gas into four proportions and squirts in parallel from the duck-billed nozzles of the four branches. A 10-mm distance between each nozzle maintains continuous cooling to prevent excessive arc heat from causing surface ablation of the nozzles during the additive process. The linear distance between nozzles and the as-deposited wall is $20 \mathrm{~mm}$ and the angle between the nozzles and the depositing wall is $20^{\circ}$. Besides, to avoid the negative effects of cooling gas on the OL process, the OL process was conducted after 6 layers. The previous 6 layers were deposited via the NC process. To verify that the cooling air did not negatively impact on the molten pool, the molten pool was monitored using a high-speed camera. From Figure 3, it is evident that there was no wandering of the arc, and the molten drop transited smoothly, the transition mode of the droplet was projected transfer and the droplet transferred to the molten pool without spatter. At the same time, the "bell jar" shaped arc remained stable. Furthermore, the WAAM process was not affected by cooling air via the OL process.

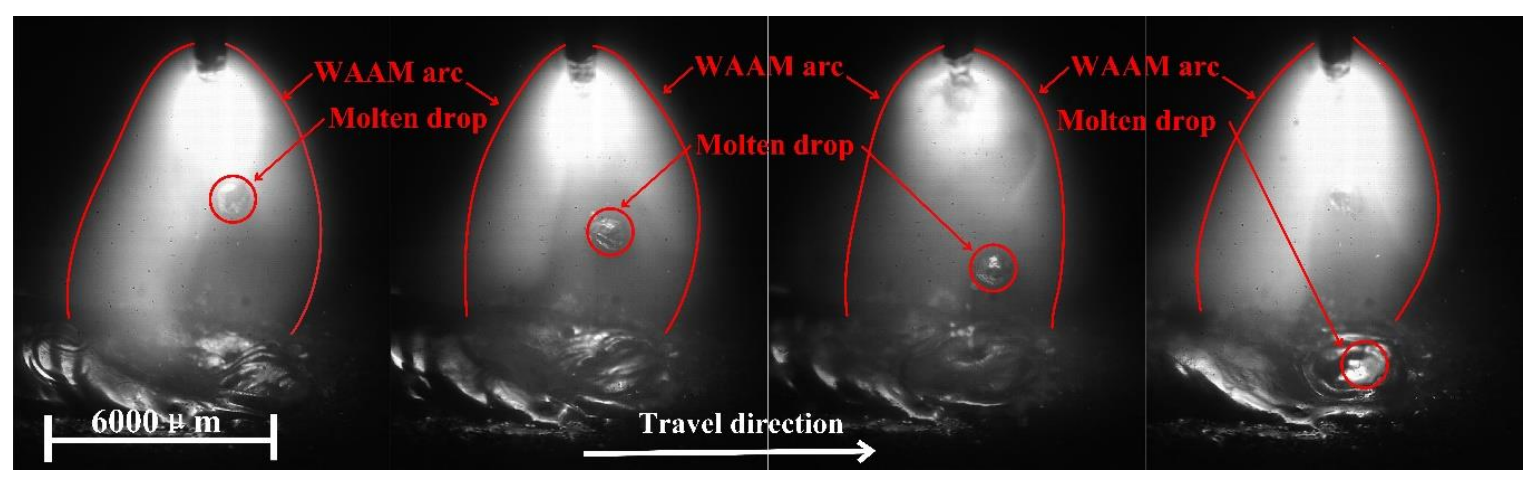

Figure 3. Image of the molten pool taken by a high-speed camera during the on-line cooling $(\mathrm{OL})$ process. 


\subsection{Porosity}

The WAAM 5356 aluminum alloy is mainly associated with porosity defects. The forming quality of the deposits could be effectively distinguished by examining the number and size of pores. In this study, we used the Olympus GX51 metallographic microscope(Olympus Inc., Shinjuku, Tokyo, Japan) to observe pore distribution, while Image-Pro Plus software(MEDIA CYBERNETICS Inc., Rockville, MD, USA) was used to measure the pore size and number in metallographic specimens; thus, the porosity of the sample was calculated.

\subsection{Material Characterization Techniques}

Both metallographic specimens and mechanical test specimens were extracted from the as-deposited 5356 walls for further assessment. The metallographic specimens were ground and polished to a mirror finish. The polish process was conducted mechanically and electrochemically. The specimens were etched for $10 \mathrm{~s}$ in an electrolyte containing $10 \%$ vol $\mathrm{HClO}_{4}$ with balance $\mathrm{C}_{2} \mathrm{H}_{5} \mathrm{OH}$, with the decomposition voltage $25 \mathrm{~V}$. Then, the specimens were etched in an anode coating solution containing $1000 \mathrm{~mL} \mathrm{H} \mathrm{H}_{2} \mathrm{O}, 30 \mathrm{~mL} \mathrm{HF}$, and $11 \mathrm{~g} \mathrm{H}_{3} \mathrm{BO}_{3}$. The anode coating process lasted for $90 \mathrm{~s}$ with the decomposition at $20 \mathrm{~V}$. The anode coated specimens were observed using an OLYMPUS GX71 optical microscope (OM) (Olympus Inc., Shinjuku, Tokyo, Japan), while the electrochemically polished specimens were observed using a ZESSIS SEGMA500 field emission scanning electron microscope (SEM) (ZESSIS Inc., Jena, Thuringia, Germany) and the electrochemically polished specimens in the SEM device were observed by an OXFORD energy dispersive spectrometer (EDS) (OXFORD INSTRUMENTS Inc., Oxford, UK). The polished specimens were observed using a MPDDY2049 X-ray diffractometer (XRD) (PANalytical B.V., Almoro, ND, USA). Vickers hardness testing was performed with an HVS-1000A automatic hardness tester (HUAYIN Inc., Laizhou, Shandong, China) using a test load of $200 \mathrm{~g}$ and a dwell time of $15 \mathrm{~s}$. Tensile tests were performed at room temperature, with a constant crosshead displacement rate of $0.2 \mathrm{~mm} / \mathrm{min}$ using an MTS370 universal testing machine (MTS Inc., Eden Prairie, MN, USA). The tensile test specimens and metallographic specimens extracted from the as-deposited walls are shown in Figure 4. According to ISO6892-1:2009, the blank parts in the size of Figure 4 was cut from the thin wall by wire-electrode cutting, and then the tensile specimen meeting the tolerance requirements of ISO6892-1:2009 was obtained by milling and grinding. ISO6892-1:2009 was used to stretch the specimen.

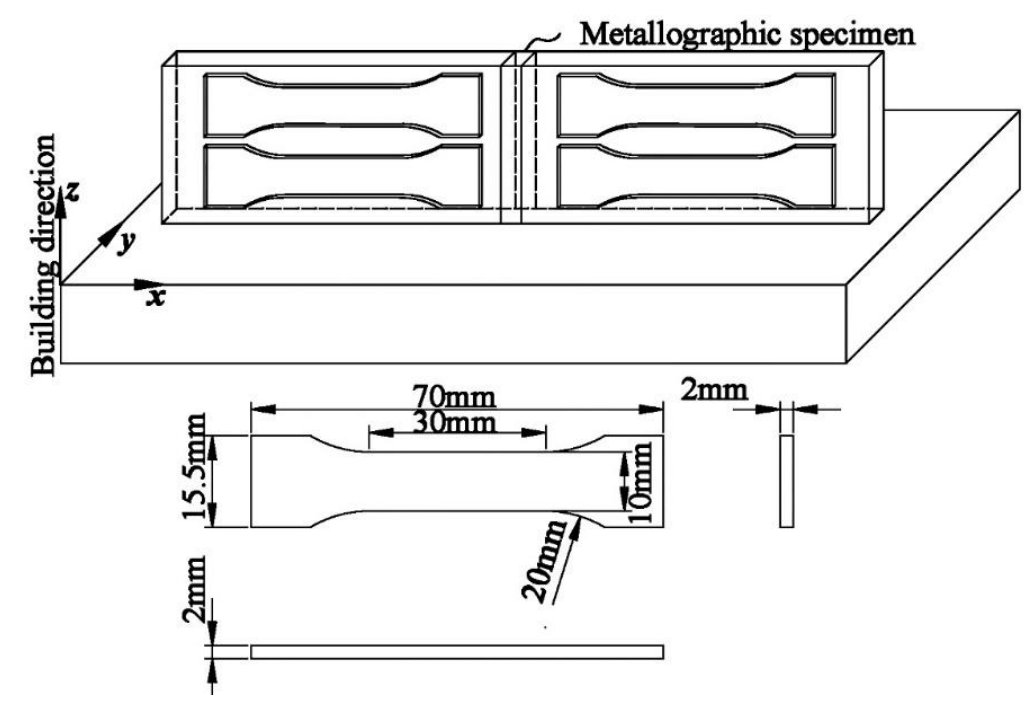

Figure 4. Schematic diagram of metallographic specimens and mechanical test specimens in as-deposited walls. 


\section{Results and Discussion}

\subsection{Geometry}

At an interlayer dwell time of $90 \mathrm{~s}$, the surface temperature was measured before depositing the next layer. The light spot size of the infrared thermometer we used was $5 \mathrm{~mm}$. We timely measured the current layer before depositing the next layer. During the measurement, we measured the temperature central position of each layer, the temperature curves obtained are shown in Figure 5. With IC, SC, and OL cooling processes, the surface temperature of the as-deposited walls tended to be stable as the number of layers increased. However, the surface temperature increased continuously with the NC process. Furthermore, with the OL process, the temperature stabilized to $30{ }^{\circ} \mathrm{C}$ (Figure 5 ), this was the first cooling process to achieve temperature stability, and the temperature dropped to a minimum compared to other cooling processes. The lower interlayer temperature implies that the deposition process can easily be controlled, Notably, the temperature was recorded after 6 layers because these layers were close to the substrate and heat mainly dissipated as a result of the substrate.

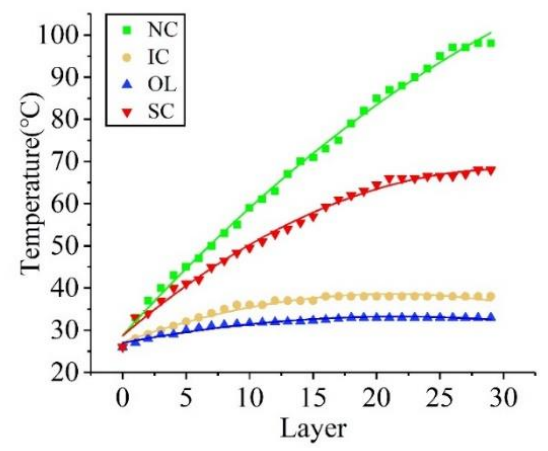

Figure 5. Temperature of each layer deposited by different cooling processes.

The dimensions of WAAM 5356 walls fabricated by different cooling processes are shown in Figure 6. Partial pits were observed at both ends of as-deposited walls and were deposited by IC, SC, and NC processes. Because heat accumulates at the ends of as-deposited walls and significantly surpasses the melting point of the previous layers, there was less support at the ends than the middle position. During the WAAM process, when the wire feed stops, an arc pit is created at the end of the motion path because the arc extinguishes without the feeding wire. No obvious arc pits were observed at either ends of the as-deposited wall that was deposited by the OL process.

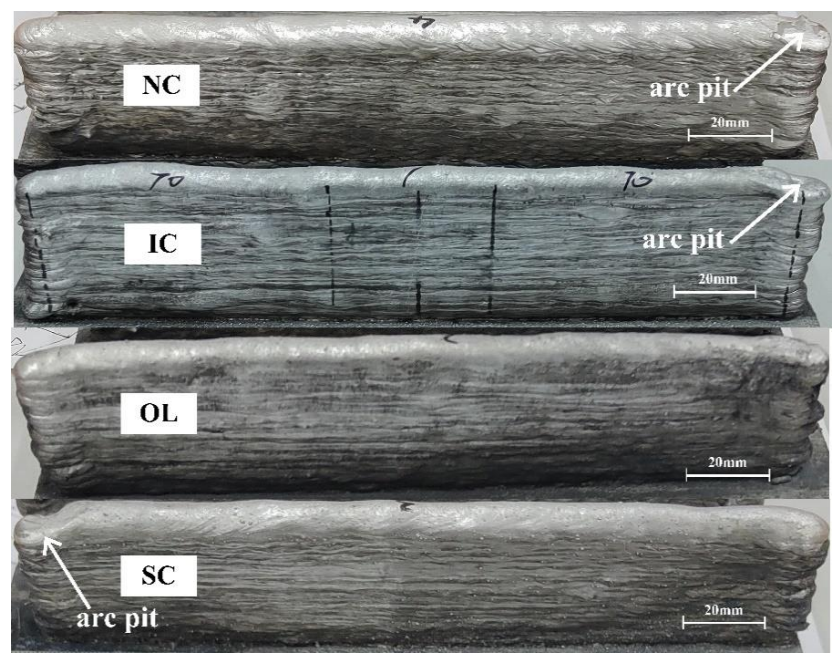

Figure 6. Geometry of WAAM as-deposited 5356 walls in different cooling processes. 
The thin wall section outline obtained by Adobe PhotoShop is highlighted in Figure 7. Of note, the section is located in the middle of the as-deposited walls. The waviness of the walled side can roughly be seen in Figure 7; however, the waviness of the as-deposited wall deposited by the OL process was significantly smaller than others, and the average thickness was smaller than with other cooling processes. The specific value of thickness change in the height direction is shown in Figure 8. From the analysis, the waviness was $0.5 \mathrm{~mm}$ via the OL process and the average thickness was $9.4 \mathrm{~mm}$, which was close to $9 \mathrm{~mm}$ of model thickness. The thickness of the as-deposited wall with the NC process did not attain a stable height direction because the temperature was continuously rising as the layer deposited. Heat accumulation was greater than heat dissipation, and the aluminium alloy was spread along via the NC process; thus, the thickness reached $10.8 \mathrm{~mm}$ from $9.4 \mathrm{~mm}$. With the increase in the layers, the temperature of the interpass with the NC process continues to rise. According to the solidification characteristics of aluminum alloy, high interlayer temperature cause a low temperature gradient, which was conducive to columnar crystal growth. At the same time, with high interlayer temperature, the aluminum alloy metal has strong fluidity and can easily produce the fusion defect at the edge of the interlayer, while the OL cooling rate is not easy to form with columnar crystal, and, because of the rapid cooling, the defective fusion cannot easily occur at the edge of interlayers.
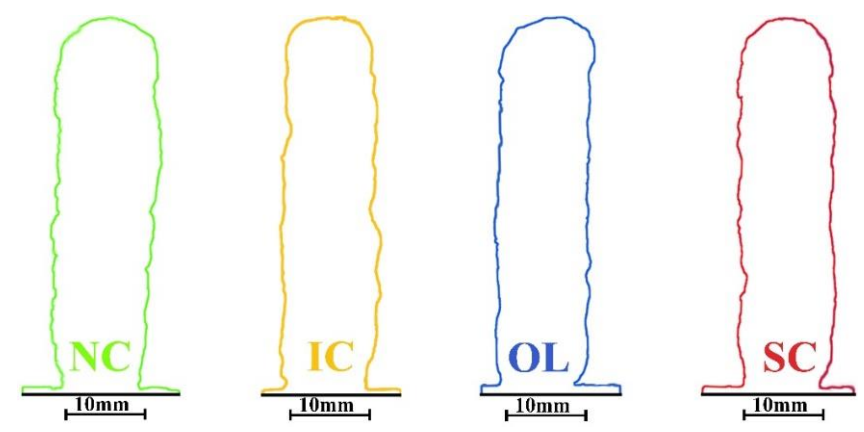

Figure 7. Section outline of as-deposited 5356 walls by different cooling processes.

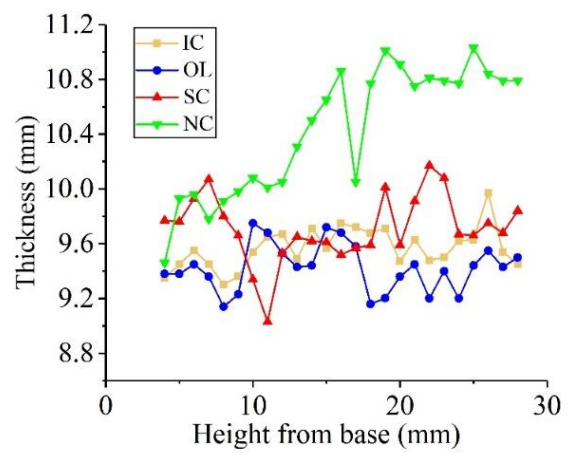

Figure 8. Thickness of as-deposited walls in the direction of height deposited by different cooling processes.

\subsection{Microstructure}

The pore defects on the cross-section of as-deposited walls are shown in Figure 9. The pores are generally distributed near the fusion line in Figure 9a. During the solidification of the molten pool, the solubility limit of the hydrogen atom decreases, $\mathrm{H}$ atoms migrate to the high solubility region because the bottom of the molten pool is the previous layer in the WAAM process. The metal close to the bottom of the molten pool solidifies faster than that at top of the molten pool, and $\mathrm{H}$ atoms escape to the top layer. As the top molten pool solidifies, $\mathrm{H}$ atoms coalesce and form hydrogen. A proportion of hydrogen evaporates into air, while a small proportion remains in the top region of the molten pool; this indicates that pores are mainly entrapped at the top of the layer [17]. Extreme pore coarsening could be observed at the edge of the as-deposited wall by the NC process (Figure 9b). Poor fusion was found at the edge of the wall, pore coarsening was caused by both hydrogen and poor fusion 
defect. In Figure 10, the porosity and maximum size of a single pore are highlighted. Pores from the NC process were exposed to higher temperatures due to the poor heat dissipation compared to the other three cooling processes; this potentially allowed hydrogen diffusion and pore coarsening. Besides, the porosity generated by the NC process was almost 8 folds higher than that generated by the OL process (the porosity via the OL process was $0.1 \%$ ). Rapid cooling promoted the diffusion rate of hydrogen atoms while it reduced the generation of hydrogen pores.
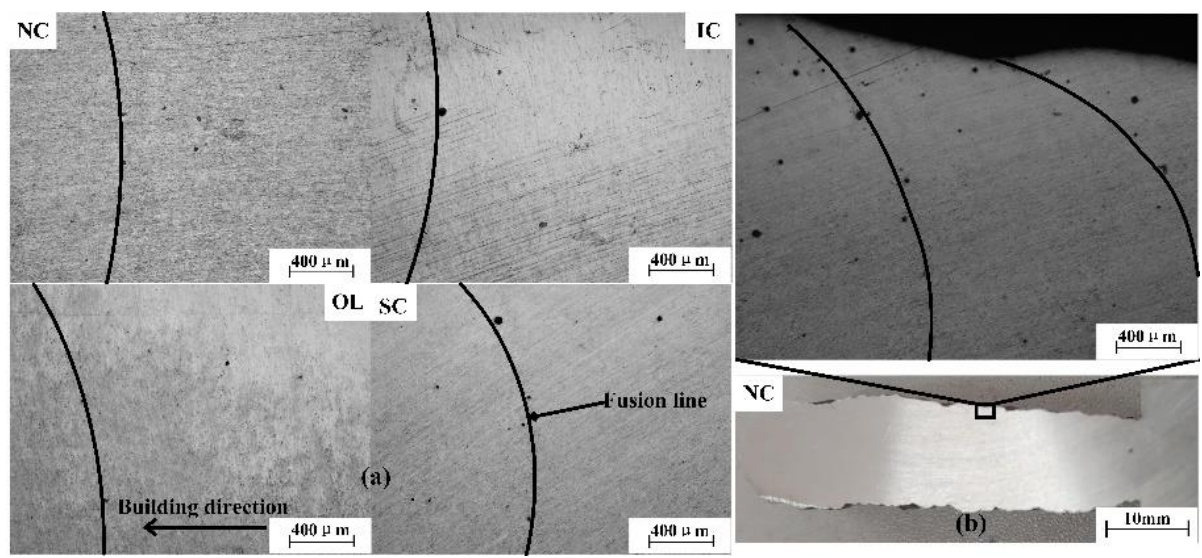

Figure 9. Defects in macroscopic morphology: (a) the pores near the fusion line; (b) pores at the edge of the wall deposited by the natural cooling (NC) process.

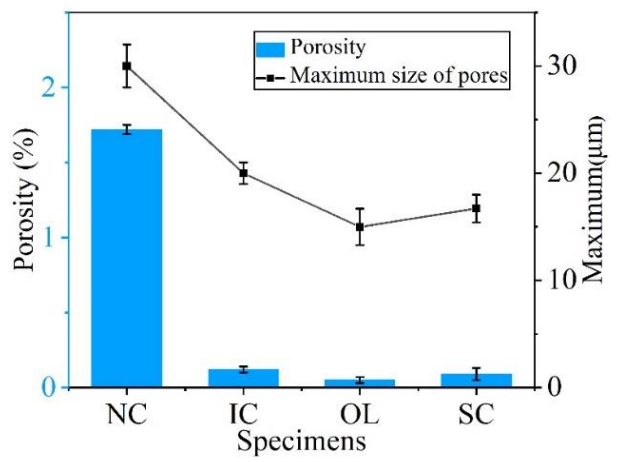

Figure 10. Porosity and maximum size of a single pore in the as-deposited specimens deposited by different cooling processes.

The interlayer grains of Al5356 alloy deposited by WAAM were observed by an optical microscope (Figure 11). The solid lines signed the fusion line, the region between two solid lines is a deposited layer, and the dotted lines represent the boundary line between columnar grains and equiaxed grains. Columnar and equiassic structures in the interlayer volumes are destroyed by recasting, and structures that are so clean and homogeneous from the metallographic point of view are difficult to create. During the solidifying process of WAAM aluminum alloy, temperature gradient $(G)$ and solidifying rate $(R)$ significantly impact the microstructure of solidification. $G$ and $R$ jointly determine the microstructure of solidification, $G / R$ determines the solidification mode, whereas $G \times R$ determines the grain size [18]. As the molten pool solidifies, there was high $G$ at the bottom of the molten pool, and the undercooling decreased, the grain morphology near the bottom edge of the molten pool was columnar, while an epitaxial growth occurred at the bottom of the molten pool. Notably, at the bottom of the molten pool, the grain of 5356 aluminum alloy (fcc), which, in the direction of $<100>$, grew rapidly into columnar grains along the direction of the temperature gradient. As the molten pool solidified, $R$-value increased while $G / R$ value decreased, and the equiaxed grain region appeared. At a stronger cooling effect, a larger $R$-value was obtained, and the $G$-value close to the bottom edge of the molten pool significantly decreased. Subsequently, the $G / R$ decreased, and the undercooling increases; thus, a large range of 
equiaxed grains are more likely to occur in an interlayer. From Figure 11c, the equiaxed grain region by the OL process was the widest $805 \mu \mathrm{m}$ among the four cooling processes. However, regional columnar grains were found (Figure 11a), which passed through the layer due to the accumulated heat via the NC process.
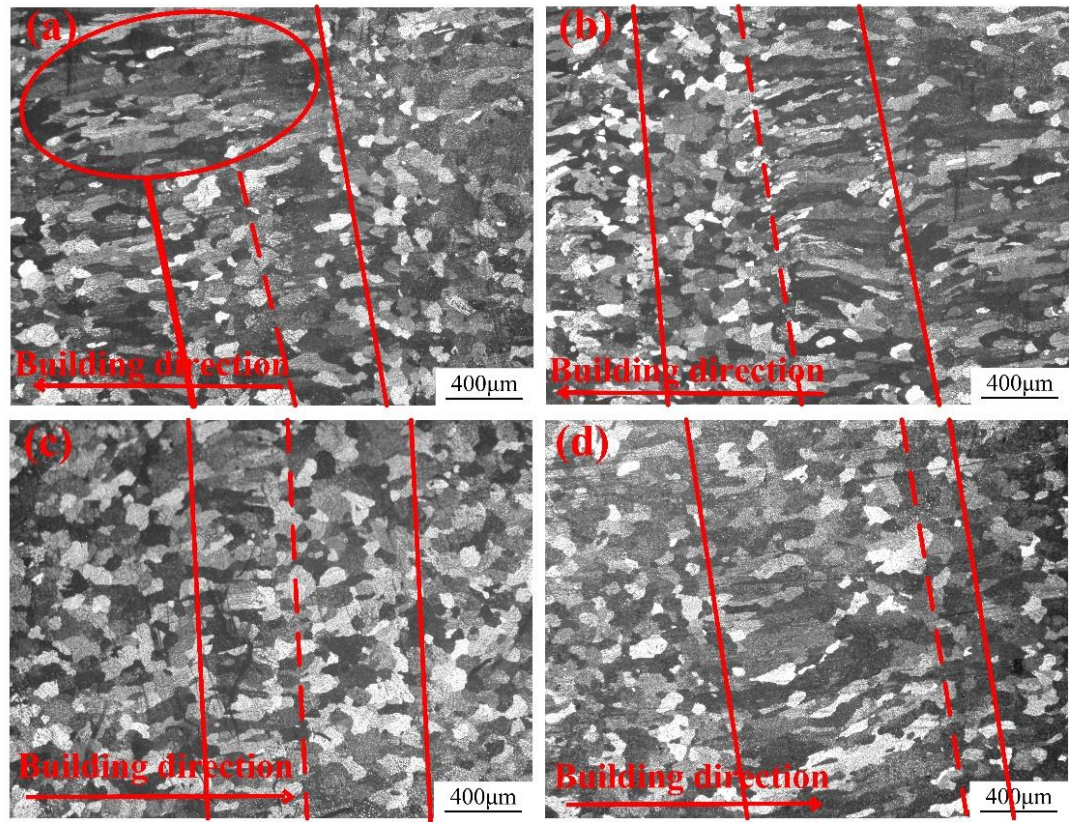

Figure 11. Optical microscope (OM) photographs of samples deposited by the (a) NC process, (b) interlayer cooling (IC) process, (c) OL process, and (d) substrate cooling (SC) process.

From XRD (Figure 12), the second phase of the deposited 5356 aluminum alloy is mainly $\beta$ $\left(\mathrm{Mg}_{2} \mathrm{Al}_{3}\right)$ phase. The microstructure morphology close to the fusion line observed by SEM is shown in Figure 13, while components of the structure obtained by EDS composition analysis are shown in Figure 14. The skeletal structure (a) was identified as the $\beta$ phase $\left(\mathrm{Al}_{2} \mathrm{Mg}_{3}\right)$ [19]. The $\beta$ phase was found near the fusion line. The non-equilibrium solidification of aluminum WAAM inevitably caused the non-uniformity of microstructure and composition. This inhomogeneity is characterized by the appearance of the non-equilibrium second phase. When the grains at the bottom of the molten pool grow into columnar grains, the first crystallized columnar grains exhibit a low solute concentration, while the later crystallized dendrites have a slightly higher solute concentration. The second phase and impurities gathered easily on the top of the molten pool [20]. Since the specimen obtained via the OL process have the fastest cooling rate, the equilibrium distribution coefficient $\mathrm{k}_{0}$ is the least. The microsegregation of OL-deposited ER5356 alloy was the most severe among the four cooling processes, but, on the whole, the distribution of elements and phases is relatively uniform between and within layers (Figure 15).

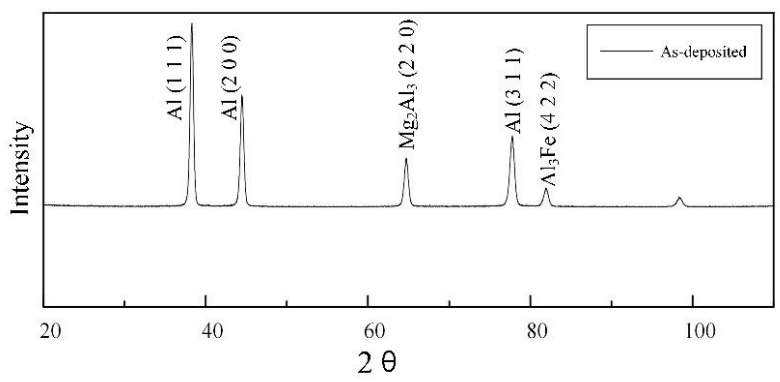

Figure 12. XRD of as-deposited walls. 


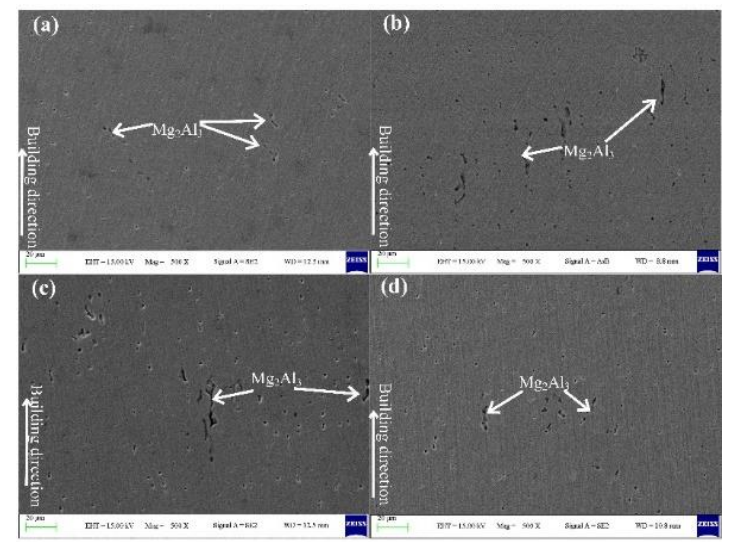

Figure 13. Scanning electron microscope (SEM) of the $\beta\left(\mathrm{Mg}_{2} \mathrm{Al}_{3}\right)$ phase in thin walls deposited by the (a) NC process, (b) IC process (c), OL process, and (d) SC process.

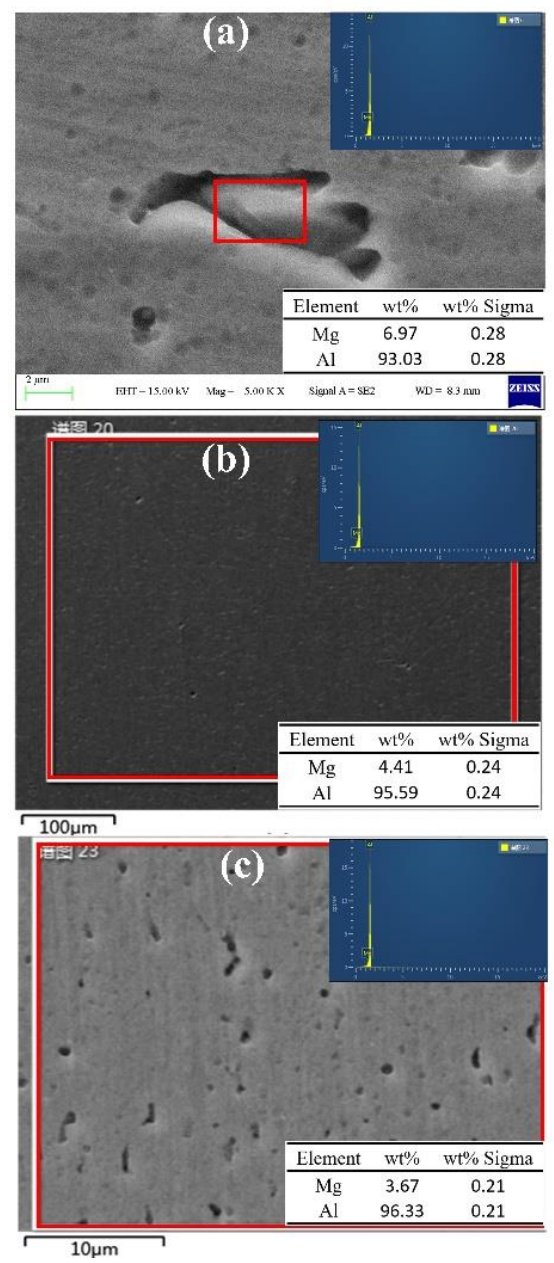

Figure 14. Energy Dispersive Spectrometer (EDS) results of the microstructure characteristics observed via a scanning electron microscope (SEM): (a) skeletal structure; (b) feeding wire; (c) specimen by NC process. 


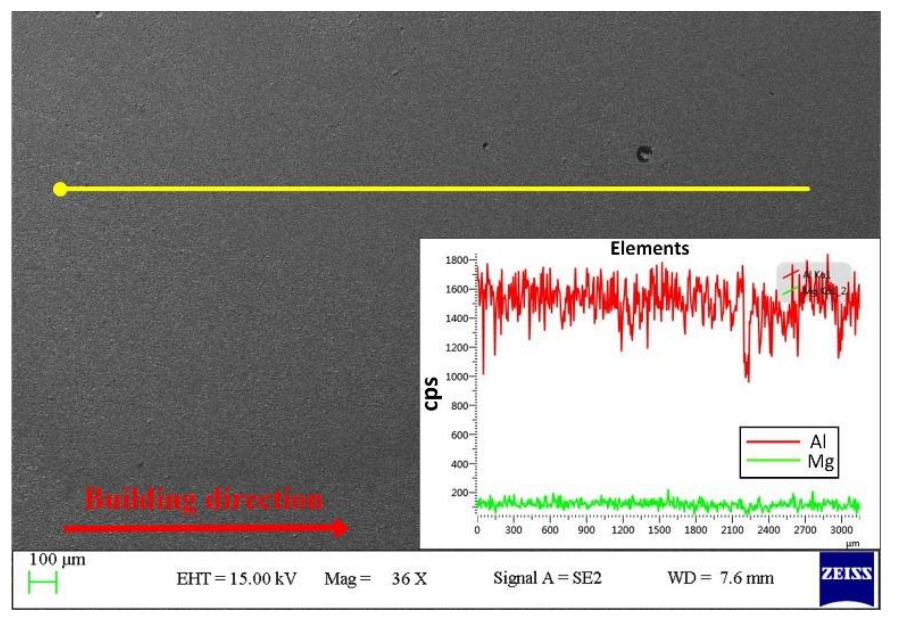

Figure 15. EDS linear scanning through layers with length of $3 \mathrm{~mm}$.

Through the EDS point scanning of samples, the Mg content of the specimen adopted via the NC process (Figure 14c) was significantly lower than that of the feeding wire materials in Figure 14b. This is attributed to the melting loss of the Mg element during the WAAM process, which consequently reduces the segregation degree.

\subsection{Mechanical Properties}

Stress-strain curves were observed during the tensile process (Figure 16). The tensile properties of the as-deposited walls are highlighted in Figure 17. The yield strength (131.8 Mpa) via the OL process slightly improved and was $12 \mathrm{Mpa}$ higher than the NC process. The strength of the alloy relies on the stresses required to overcome obstacles in dislocation motion, these obstacles are defects such as solute atoms, precipitates, other dislocations, and grain boundaries. Thus, the yield strength $\left(\sigma_{y}\right)$ of the as-deposited specimen defines pure aluminum $\left(\sigma_{y 1}\right)$ and different defects $\left(\Delta \sigma_{i}\right)$ to its mechanical property, as follows:

$$
\begin{gathered}
\sigma_{y}=\sigma_{y 1}+\Delta \sigma \\
\Delta \sigma=\Delta \sigma_{h p}+\Delta \sigma_{s}+\cdots
\end{gathered}
$$

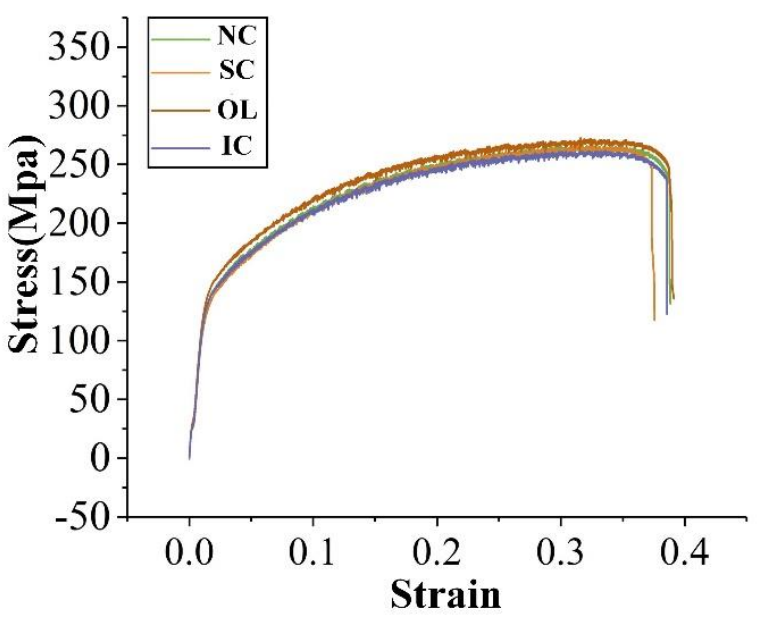

Figure 16. Stress-strain curves of the fourth cooling process. 


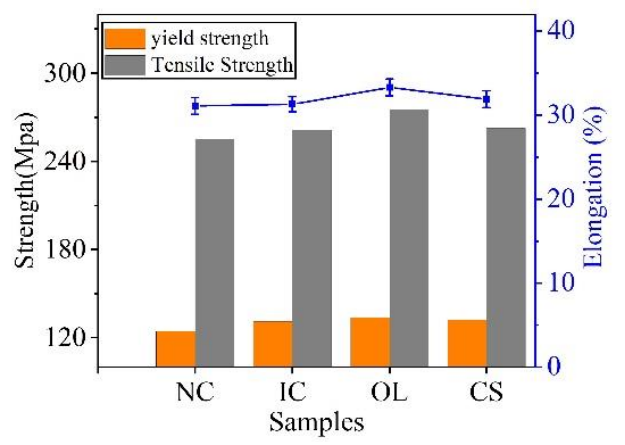

Figure 17. Yield strength (Mpa), tensile strength (Mpa), and elongation (\%) of as-deposited specimens by different cooling processes.

In this study, due to the significant effect of the fast cooling rate on grain size by the OL process, as shown in Figure 11, a large equiaxed grains area occurred. Thus, a small average grain size of the as-deposited specimen was present. The reduction in grain size increases the yield strength according to Hall-Petch relation, as follows:

$$
\Delta \sigma_{h p}=K d^{-0.5}
$$

where $d$ is the average grain diameter and $K$ is a constant [21]. Based on Equation (3), increasement of $\Delta \sigma_{h p}$ was predicted owing to the effects of grain refinement.

Due to the high cooling rate produced by the OL process, as-deposited Al material in most cases does not contain coarse intermetallic particles. However, we found a small amount of Mg-rich precipitate in the specimens (Figure 13), although the volume fraction was slightly low. This was an indication that $\mathrm{Mg}$ mainly exists in the molten pool as a supersaturated molten solution, and the as-deposited specimens were not strengthened by the Orowan process. Mg atoms dissolved in the matrix $\mathrm{Al}$ form point defects that impede dislocation motion. Moreover, $\mathrm{Mg}$ atoms diffuse near the moving dislocation during loading [22,23]. Thus, dislocation motion was suppressed during loading and $\Delta \sigma_{s}$ was increased which is defined as follows:

$$
\Delta \sigma_{s}=H C^{\alpha}
$$

where $C$ is the $\mathrm{Mg}$ concentration (wt. \%) and $\alpha$ and $H$ are constants [24]. The $\alpha$ and $H$ measured the strain rate. Therefore, the $\Delta \sigma_{s}$ was determined by the amount $(C)$ of $\mathrm{Mg}$ in the matrix $\mathrm{Al}$ (Figure 14). Based on Equation (4), because of the melting loss of the Mg element was extreme during the NC process, the decrease was predicted owing to the effects of the amount $(C)$ of $\mathrm{Mg}$ in the matrix $\mathrm{Al}$ with the NC process. Of note, the strength of as-deposited specimens was partly attributable to grain refinement and $\mathrm{Mg}$ concentration. Because tensile strength is affected by yield strength, the variation between them is the same, as shown in Figure 17.

The highest elongation occurred by the OL process (Figure 17); this can be explained by the grain refinement. That is, in a definite volume of an as-deposited specimen and under the same amount of plastic deformation, with the formation of equiaxic grains, the defective state was restored by the OL process; thus, cracking caused by stress at concentration opportunity was less and showed higher plasticity than other cooling processes. Similarly, in Figure 18, the highest microhardness through the OL process is depicted; this can further be explained by the grain refinement, which caused a significant increase in grain boundaries. The array of atoms in the interface was uneven and a considerable amount of defects occurred, this obstructed the dislocations; thus, the hardness increased. 


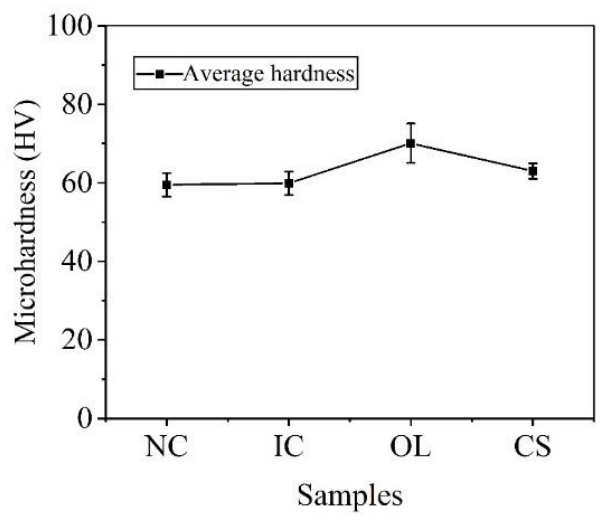

Figure 18. Microhardness (HV) of as-deposited specimens via different cooling processes.

The fracture section morphology of the tensile specimens is shown in Figure 19. All the tensile samples fractured within the gauge length, and the obvious necking occurred in fracture position. Figure 19a shows the fracture morphology of the specimen deposited by the NC process, while Figure 19b shows the fracture morphology by the OL process. Several dimples and microscopic holes are distributed in the (b3) fracture, which reveals obvious ductile fracture characteristics. The existence of the second phase particles led to the final fracture, which showed a typical transgranular fracture and formed a dimple fracture morphology. The intergranular fracture characteristic (a2) was found in (a1) and was closely associated with the NC process, resulting in the columnar grain size. Moreover, the obvious intergranular fracture is caused by coarse grains, affecting the plasticity and other mechanical properties of the formed sample.

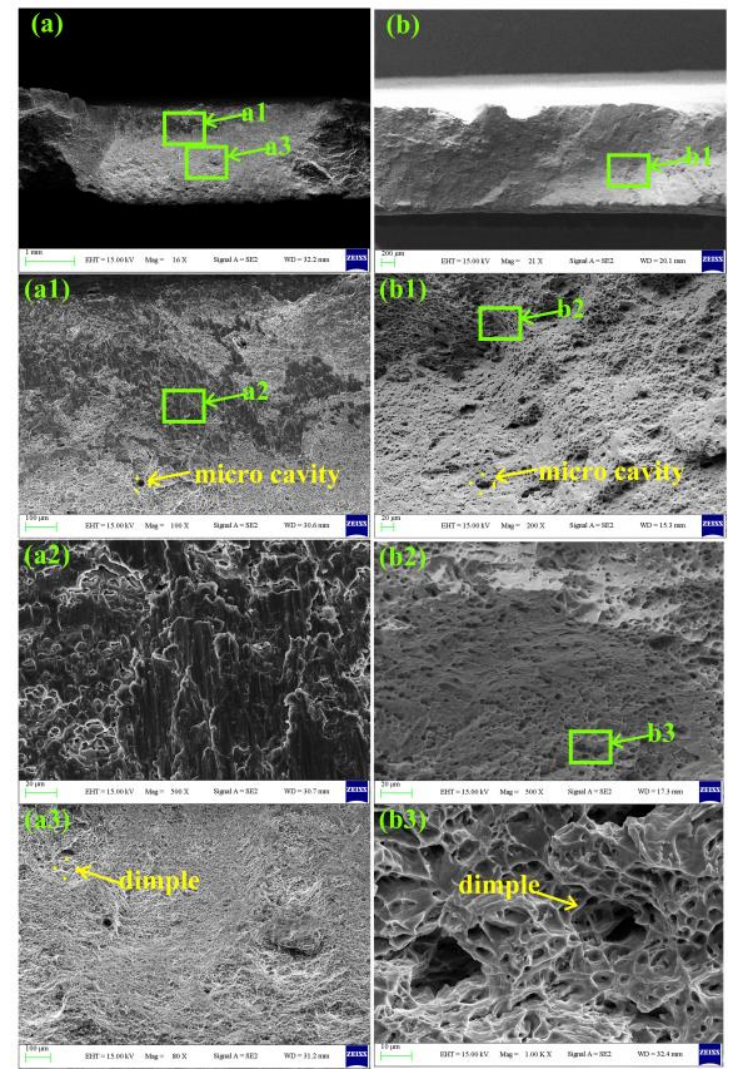

Figure 19. Tensile fracture at room temperature: (a) deposited by the NC process; (b) deposited by the OC process. 


\section{Conclusions}

In this study, three different cooling processes were conducted in the WAAM 5356 alloys. The effects of different cooling processes on the geometry forming, defect, metallography, and properties were investigated. The major findings include:

In the WAAM process for the Al-Mg alloy, the adoption of the OL process maintained a minimum interlayer temperature, and the as-deposited wall thickness was the easiest to control to a specified size; in particular, the arc pit at the end of the wall was not visible which improved the stability of the WAAM process.

The porosity rate was reduced to $0.1 \%$ with the OL process, and a decrease in the interpass temperature during the WAAM process had a significant impact on grain refinement. The EDS and XRD analysis of the microstructure revealed that the $\beta$ phase mainly appeared near the fusion line, and the interlayer segregation was most serious with the OL process, and the magnesium element that burned out occurred via the NC process.

It was evident that cooling regulation improved the yield strength, plasticity, and hardness, among which, the OL process had the best effect. Fine-grained strengthening plays an important role in the OL method. Although the tensile specimens prepared via different cooling processes were plastic fracture, the NC specimen shows the possibility of partial cleavage fracture.

Author Contributions: Conceptualization, S.Z., G.Y. and B.W.; Data curation, G.Y. and B.W.; formal analysis, G.Y.; Funding acquisition, C.C. and L.Q.; investigation, B.W. and C.C.; Methodology, B.W., S.Z. and G.Y.; project administration, L.Q. and C.C.; validation, G.Y., S.Z. and L.Q.; visualization, B.W. and G.Y.; writing-review and editing, B.W. and S.Z. All authors have read and agreed to the published version of the manuscript.

Funding: This study was funded by the Rapid Response Project of the Special Zone of National Defense of Science and Technology Commission of CMC (NO. 18-H863-31-ZD-003-001-0) and National Natural Science Foundation of China (NSFC) (NO. 51975387).

Acknowledgments: This study was supported by the Rapid Response Project of the Special Zone of National Defense of Science and Technology Commission of CMC (NO. 18-H863-31-ZD-003-001-0) and National Natural Science Foundation of China (NSFC) (NO. 51975387).

Conflicts of Interest: The authors declare no conflict of interest.

\section{References}

1. Rodrigues, T.A.; Duarte, V.; Miranda, R.M.; Santos, T.G.; Oliveira, J.P. Current Status and Perspectives on Wire and Arc Additive Manufacturing (WAAM). Materials 2019, 12, 1121. [CrossRef] [PubMed]

2. Wang, Y.; Chen, X.; Konovalov, S.V. Additive Manufacturing Based on Welding Arc: A low-Cost Method1. J. Surf. Investig. X-ray Synchrotron Neutron Tech. 2017, 6, 1317-1328. [CrossRef]

3. Shamsaei, N.; Yadollahi, A.; Bian, L.; Thompson, S.M. An overview of Direct Laser Deposition for additive manufacturing; Part II: Mechanical behavior, process parameter optimization and control. Addit. Manuf. 2015, 8, 12-35. [CrossRef]

4. Kruth, J.P.; Froyen, L.; Van Vaerenbergh, J.; Mercelis, P.; Rombouts, M.; Lauwers, B. Selective laser melting of iron-based powder. J. Mater. Process. Technol. 2004, 149, 616-622. [CrossRef]

5. Williams, S.W.; Martina, F.; Addison, A.C.; Ding, J.; Pardal, G.; Colegrove, P. Wire + Arc Additive Manufacturing. Mater. Sci. Technol.-Lond. 2016, 7, 641-647. [CrossRef]

6. Colegrove, P.; Williams, S. High Deposition Rate High Quality Metal Additive Manufacture Using Wire + Arc Technology; Cranfield University: Bedford, UK, 30 December 2013; pp. 1-42.

7. Zhang, C.; Li, Y.; Gao, M.; Zeng, X. Wire arc additive manufacturing of Al-6Mg alloy using variable polarity cold metal transfer arc as power source. Mater. Sci. Eng. A 2018, 711, 415-423. [CrossRef]

8. Derekar, K.; Lawrence, J.; Melton, G.; Addison, A.; Zhang, X.; Xu, L. Influence of Interpass Temperature on Wire Arc Additive Manufacturing (WAAM) of Aluminium Alloy Components. MATEC Web Conf. 2019, 269, 5001. [CrossRef]

9. Ren, L.; Gu, H.; Wang, W.; Wang, S.; Li, C.; Wang, Z.; Zhai, Y.; Ma, P. Effects of Interpass Cooling on Material Properties of Wire Arc Additive Manufactured Al-6.3Mg Alloy. 3D Print. Addit. Manuf. 2019, 6, 344-353. [CrossRef] 
10. Geng, H.; Li, J.; Xiong, J.; Lin, X. Optimisation of interpass temperature and heat input for wire and arc additive manufacturing 5A06 aluminium alloy. Sci. Technol. Weld. Join. 2017, 22, 472-483. [CrossRef]

11. Henckell, P.; Günther, K.; Ali, Y.; Bergmann, J.P.; Scholz, J.; Forêt, P. The Influence of Gas Cooling in Context of Wire Arc Additive Manufacturing-A Novel Strategy of Affecting Grain Structure and Size. In Proceedings of the TMS 2017 146th Annual Meeting \& Exhibition Supplemental Proceedings, San Diego, CA, USA, 30 March 2017; Society, M.M., Ed.; Springer: Cham, Switzerland; San Diego, CA, USA, 2017; pp. 147-156.

12. Henckell, P.; Gierth, M.; Ali, Y.; Reimann, J.; Bergmann, J.P. Reduction of Energy Input in Wire Arc Additive Manufacturing (WAAM) with Gas Metal Arc Welding (GMAW). Materials 2020, 13, 2491. [CrossRef]

13. Su, C.; Chen, X.; Gao, C.; Wang, Y. Effect of heat input on microstructure and mechanical properties of Al-Mg alloys fabricated by WAAM. Appl. Surf. Sci. 2019, 486, 431-440. [CrossRef]

14. Wu, B.; Pan, Z.; Ding, D.; Cuiuri, D.; Li, H. Effects of heat accumulation on microstructure and mechanical properties of Ti6Al4V alloy deposited by wire arc ad-ditive manufacturing. Addit. Manuf. 2018, 23, 151-160. [CrossRef]

15. Wu, B.; Pan, Z.; Ding, D.; Cuiuri, D.; Li, H.; Fei, Z. The effects of forced interpass cooling on the material properties of wire arc additively manufactured Ti6Al4V alloy. J. Mater. Process. Technol. 2018, 258, 97-105. [CrossRef]

16. Li, F.; Chen, S.; Shi, J.; Zhao, Y.; Tian, H. Thermoelectric Cooling-Aided Bead Geometry Regulation in Wire and Arc-Based Additive Manufacturing of Thin-Walled Structures. Appl. Sci. 2018, 8, 207. [CrossRef]

17. Gu, J.; Ding, J.; Williams, S.W.; Gu, H.; Ma, P.; Zhai, Y. The effect of inter-layer cold working and post-deposition heat treatment on porosity in additively manufactured aluminum alloys. J. Mater. Process. Technol. 2016, 230, 26-34. [CrossRef]

18. Mullins, W.W.; Sekerka, R.F. Stability of a Planar Interface during Solidification of a Dilute Binary Alloy. J. Appl. Phys. 1964, 35, 444-451. [CrossRef]

19. Okamoto, H. Al-Mg (aluminum-magnesium). J. Phase Equilibria Diffus. 1998, 6, 598. [CrossRef]

20. Bai, J.Y.; Fan, C.L.; Lin, S.B.; Yang, C.L.; Dong, B.L. Effects of thermal cycles on microstructure evolution of 2219-Al during GTA-additive manufacturing. Int. J. Adv. Manuf. Technol. 2016, 87, 2615-2623. [CrossRef]

21. Thangaraju, S.; Heilmaier, M.; Murty, B.S.; Vadlamani, S.S. On the Estimation of True Hall-Petch Constants and Their Role on the Superposition Law Exponent in Al Alloys. Adv. Eng. Mater. 2012, 14, 892-897. [CrossRef]

22. Dieter, G.E.; Bacon, D.J. Mechanical Metallurgy, 1st ed.; McGraw-Hill: New York, NY, USA, 1986; Volume 1, p. 609.

23. Hull, D.; Bacon, D.J. Introduction to Dislocations, 4th ed.; Butterworth-Heinemann: Woburn, UK, 2001; Volume 1, p. 250.

24. Ryen, Ø.; Nijs, O.; Lander, E.S.; Holmedal, B.; Ekström, H.E.; Nes, E. Strengthening Mechanisms in Solid Solution Aluminum Alloys. Metall. Mater. Trans. A 2006, 6, 1999-2006. [CrossRef]

(C) 2020 by the authors. Licensee MDPI, Basel, Switzerland. This article is an open access article distributed under the terms and conditions of the Creative Commons Attribution (CC BY) license (http://creativecommons.org/licenses/by/4.0/). 